\title{
Pore-Scale Mixing and the Evolution of Hydrodynamic Dispersion in Porous Media
}

\author{
Alexandre Puyguiraud $\odot$, Philippe Gouze $\odot$, and Marco Dentz $\odot^{*}$ \\ Spanish National Research Council (IDAEA-CSIC), 08034, Barcelona, Spain and Geoscience Montpellier, \\ CNRS, Université de Montpellier, 34090, Montpellier, France
}

(Received 10 December 2020; revised 9 February 2021; accepted 24 March 2021; published 19 April 2021)

\begin{abstract}
We study the interplay of pore-scale mixing and network-scale advection through heterogeneous porous media, and its role for the evolution and asymptotic behavior of hydrodynamic dispersion. In a Lagrangian framework, we identify three fundamental mechanisms of pore-scale mixing that determine large scale particle motion, namely, the smoothing of intrapore velocity contrasts, the increase of the tortuosity of particle paths, and the setting of a maximum time for particle transitions. Based on these mechanisms, we derive a theory that predicts anomalous and normal hydrodynamic dispersion in terms of the characteristic pore length, Eulerian velocity distribution, and Péclet number.
\end{abstract}

DOI: 10.1103/PhysRevLett.126.164501

Transport of dissolved substances through porous media is determined by the complexity of the velocity field in the pore space and diffusive mass transfer within and between pores. The interplay of diffusive pore-scale mixing and spatial flow variability are key for the understanding of transport and reaction phenomena in natural and engineered porous media [1-3] with diverse applications ranging from groundwater contamination and geological carbon dioxide storage [4], to the design of batteries [5] and transport in brain microcirculation [6].

Therefore, hydrodynamic transport has been the focus of research over decades in different disciplines [1,2,7-10]. Still, as outlined in the following, questions of fundamental nature remain concerning both the evolution of hydrodynamic dispersion, and the dependence of asymptotic hydrodynamic dispersion coefficients on the Péclet number, which compares the diffusion and advection times over a typical pore length. Anomalous dispersion phenomena [11] have been observed in laboratory experiments [12-17], field scale tracer tests [18,19], and numerical simulations in different types of porous medium and rock structures [20-26]. They cannot be described by a single constant hydrodynamic dispersion coefficient. The asymptotic concept of hydrodynamic dispersion models particle displacements due to velocity fluctuations as Brownian motion and thus implicitly assumes that all particles have access to the full fluctuation spectrum at each moment, that is, they can be considered as statistically equal. In a porous medium, however, velocities vary on length scales engraved in the pore structure, and thus, particle transitions over regions of low velocity can be much longer than over regions of high velocity. Statistical equivalence can only be achieved at times larger than the largest transition timescale. Thus, anomalous behaviors can be traced back to broad distributions of mass transfer timescales related to wide spectra of pore-scale flow velocities.
This phenomenology lies at the heart of nonlocal transport theories such as multitrapping and continuous time random walk (CTRW), which have been used to model anomalous and intermittent pore-scale transport behaviors [17,20,22,24,27-30]. However, current approaches are limited to purely advective transport, or need to be constrained by the measurement of particle transition times. The quantitative relation between pore-scale mixing, network scale flow and the evolution of hydrodynamic dispersion remains elusive. The pioneering works of de Josselin de Jong [7] and Saffman [8] use the concept of particle transition times to derive expressions for the asymptotic hydrodynamic dispersion coefficients. Still, and in spite of numerous theoretical and numerical studies [31-33], the dependence of hydrodynamic dispersion on the Péclet number remains an open issue.

We address these fundamental questions by identifying and quantifying the key mechanisms of pore-scale mixing and network-scale flow variability in a stochastic model for the prediction of hydrodynamic dispersion. We derive a theory that explains the temporal evolution of dispersion and the dependence of its asymptotic behavior on the Péclet number based on the Eulerian flow statistics, diffusion, and the characteristic velocity length scale. The theoretical developments are supported and validated by direct numerical simulations (DNS) of flow and transport in a three-dimensional digitized Berea sandstone sample (Fig. 1) obtained using x-ray microtomography [34]. The medium displays strong pore-scale heterogeneity that gives rise to a broad distribution of flow speeds illustrated in Fig. 2(a). We consider transport at different Péclet numbers, which are varied by changing the average flow rate. The molecular diffusion coefficient is set equal to $D_{m}=10^{-9} \mathrm{~m}^{2} / \mathrm{s}$. The Péclet number is here defined by $\mathrm{Pe}=\left\langle v_{e}\right\rangle \ell_{0} / D_{m}$, where $\ell_{0}$ is the average pore length and $\left\langle v_{e}\right\rangle$ the average Eulerian flow speed. 


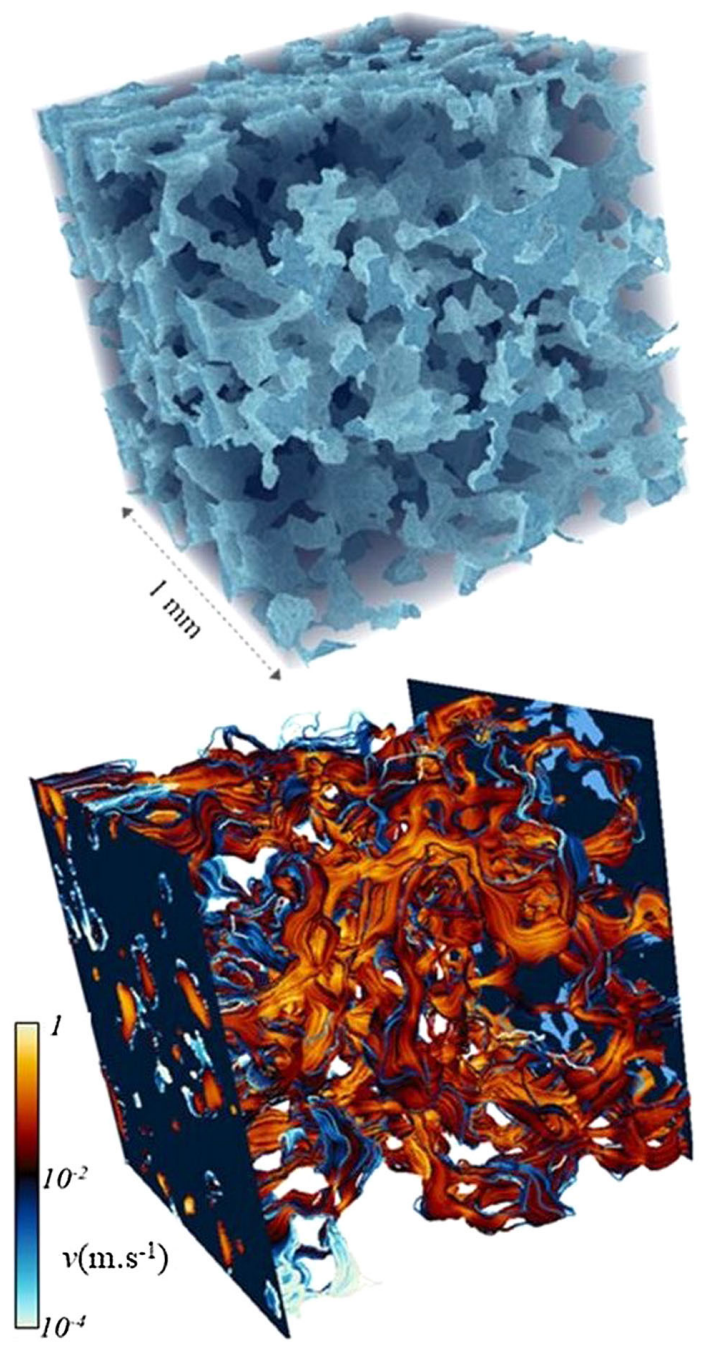

FIG. 1. Three-dimensional structure of a Berea sandstone sample. The top panel highlights the void space in shades of blue. The lines in the bottom panel show particle paths. The color scheme indicates particle speeds from (white-blue-black-orange) low to high.

Figure 3 illustrates the impact of flow heterogeneity and diffusion at different Pe. It shows on the one hand anomalous hydrodynamic dispersion manifest in heavytailed arrival time distributions $f(t)$ and superdiffusive growth of the longitudinal displacement variance $\sigma^{2}(t)$, and on the other hand cross-overs to asymptotically Fickian behaviors [Figs. 3(a) and 3(b)]. Streamwise dispersion in the asymptotic regime is characterized by the constant hydrodynamic dispersion coefficient $D^{*}$, whose nonlinear dependence on the Péclet number is shown in Fig. 3(c). These features, which result from the complex interplay of flow heterogeneity, diffusion, and geometry are generally observed in laminar flows through porous media and networks [20].

In order to understand the mechanisms that cause these behaviors, we consider particle motion along the tortuous paths in the void space of a porous medium as illustrated in
Fig. 1. The flow speed along streamlines varies over the correlation scale $\ell_{v}$ imprinted in the medium geometry and flow structure $[15,44] ; \ell_{v}$ is typically larger than the geometric pore length $\ell_{0}$ due to the tortuosity of the streamlines. We model motion along particle pathways through discrete spatial steps along conducts of length $\ell_{v}$ such that subsequent particle speeds $\left\{v_{n}\right\}$ and therefore transition times $\left\{\tau_{n}\right\}$ along a path can be considered as independent random variables. The distance $s_{n}$ and transport time $t_{n}$ of a particle along a tortuous pathway are described by the stochastic process

$$
s_{n+1}=s_{n}+\ell_{v}, \quad t_{n+1}=t_{n}+\tau_{n} .
$$

The travel distance $s(t)$ along a particle path in this coarsegrained picture is given by $s(t)=s_{n_{t}}$, where $n_{t}=\max \left(n \mid t_{n} \leq t\right)$. This picture is equivalent to representing the pore space as a network of connected conducts of length $\ell_{v}$ and average flow speeds $v_{m}$ [8], whose intersections correspond to the turning points of the process (1).

For purely advective transport, particles move along streamlines by the local Eulerian flow speed. Motion along streamlines is projected onto streamwise motion by advective tortuosity $\chi_{a}=\left\langle v_{e}\right\rangle / \bar{u}$, which is equal to the ratio between the average Eulerian flow speed $\left\langle v_{e}\right\rangle$ and average streamwise flow velocity $\bar{u}[29,45,46]$. In the presence of pore-scale diffusion this is different. First, during a transition over a conduct, particles sample the flow speeds across streamlines. Thus, the actual particle speed is different from the local flow speed along a streamline. Second, diffusion sets a maximum transition time. In fact, if the local advective particle speed is smaller than $D_{m} / \ell_{v}$, the transition time is diffusion dominated with a maximum of the order of $\tau_{D}=\ell_{v}^{2} / D_{m}$. Third, pore-scale mixing increases the effective path length and thus tortuosity.

In order to quantify these mechanisms, we first consider the flow speeds by which particles move along the conducts of length $\ell_{v}$. To this end, we assume that flow within a three-dimensional conduct of length $\ell_{v}$ can be described by the Poiseuille law, that is, by a parabolic velocity profile. This is a valid approximation because laminar flow in the porous medium is dominated by the drag due to the solid walls. If the diffusion time across is smaller than the advection time along the conduct, a particle samples the velocity profile across the conduct and moves effectively with the average conduct velocity. This is the case for narrow conducts associated with low flow velocities. For wide conducts, diffusion removes particles from the low velocities at the grain walls such that the average particle speed is increased towards the mean. Thus, particle motion along a conduct is dominated by the mean speed $v_{m}$, whose probability density function (PDF) $p_{m}(v)$ is related to the Eulerian speed PDF $p_{e}(v)$ by 


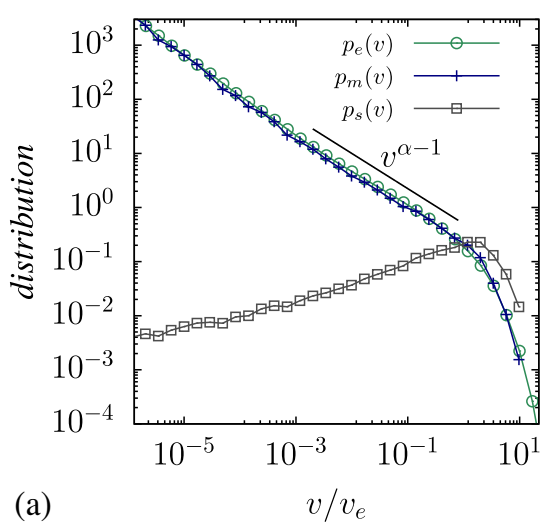

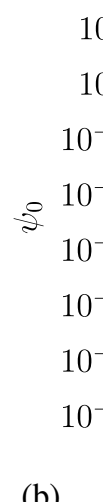

(b)

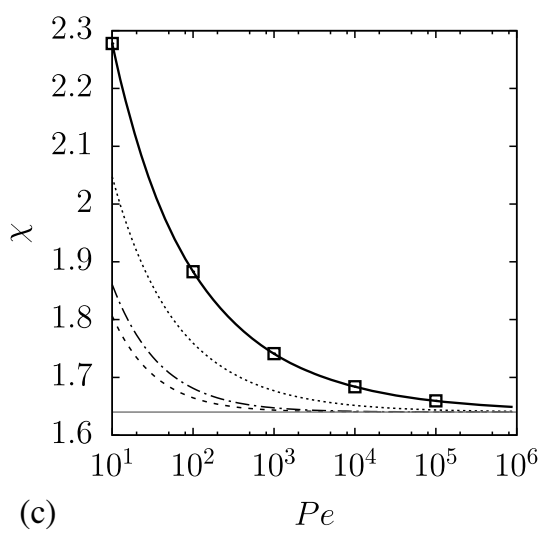

FIG. 2. (a) PDFs of (circles) Eulerian flow speed $p_{e}(v)$, (crosses) mean flow speed $p_{m}(v)$ and (squares) flux weighted mean speed distribution $p_{s}(v)$ normalized by the Eulerian mean speed $\left\langle v_{e}\right\rangle$ for the Berea sample of Fig. 1 . The solid line denotes the power-law $v^{\alpha-1}$ with $\alpha=0.35$. (b) First passage time distribution $\psi_{0}(t \mid v)$ for local Péclet numbers of (crosses) $\mathrm{Pe}_{v}=10^{2}$ (circles) 10 , (squares) 1 and (diamonds) $10^{-1}$. (c) Tortuosity $\chi$ versus Pe for Gamma distributed $p_{e}(v)$ with exponents (solid line) $\alpha=0.35$, (dotted) 0.5 , (dashdotted) 0.8 , and (dashed) 1 . The horizontal line indicates the advective tortuosity $\chi_{a}$.

$$
p_{e}(v)=\int_{0}^{\infty} d v^{\prime} p_{m}(v) \frac{1}{2 v^{\prime}} H\left(2 v^{\prime}-v\right) .
$$

The assumption of Poiseuille flow gives a uniform speed PDF in the conduct, such that the Eulerian PDF can be constructed as the weighted sum of the local uniform PDFs represented by the Heaviside function under the integral. The Eulerian PDF $p_{e}(v)$ and thus $p_{m}(v)$ are determined by volumetric sampling of the local flow speeds, while the partitioning of particles at turning points is proportional to the flow rates into the downstream conducts. Thus, the probability $p_{s}(v)$ for a particle to choose the speed $v$ at a turning point is given by the flux-weighted PDF of mean speeds [34],

$$
p_{s}(v)=\frac{v p_{m}(v)}{\left\langle v_{m}\right\rangle},
$$

where $\left\langle v_{m}\right\rangle$ is the network averaged mean velocity. Figure 2(a) displays the Eulerian $p_{e}(v)$, and $p_{m}(v)$ for the Berea sample, and the corresponding Lagrangian $p_{s}(v)$. The PDF $p_{m}(v)$ of mean speeds is very similar to $p_{e}(v)$ because of the power-law behavior at small velocities. Equations (2)-(3) provide the bridge between Eulerian and Lagrangian flow characteristics. Current approaches that explore mapping the conduct widths to flow speeds via the Poiseuille law and local mass conservation [28,47-49] provide promising avenues to ultimately relate $p_{e}(v)$ to the medium structure, which, however, is beyond the scope of this Letter.

Next we consider the impact of diffusion on the PDF of transition times over a single conduct. It is obtained by considering advective-diffusive transport in a $d=1$ dimensional domain of length $\ell_{v}$ characterized by an instantaneous injection at the upstream and an absorbing boundary condition at the downstream end [34]. The transition time $\operatorname{PDF} \psi_{0}(t \mid v)$ for a single conduct is given by the solute flux over the downstream boundary. It is sharply peaked around the advection time $\tau_{v}=\ell_{v} / v$ for high local Péclet numbers $\mathrm{Pe}_{v}=v \ell_{v} / 2 D$, and broadly distributed with an exponential cutoff at the diffusion time $\tau_{D}$ as illustrated in Fig. 2(b) for $\mathrm{Pe}_{v}=10^{2}, 10,1$, and $10^{-1}$. The mean transition time is given by [34]

$$
\langle\tau \mid v\rangle=\tau_{v} \frac{\mathrm{Pe}_{v}-\exp \left(-\mathrm{Pe}_{v}\right) \sinh \left(\mathrm{Pe}_{v}\right)}{\mathrm{Pe}_{v}} .
$$

At high $\mathrm{Pe}_{v}$ it tends to the local advection time $\tau_{v}$, at low $\mathrm{Pe}_{v}$ to $\tau_{D} / 2$. The network-scale PDF $\psi(t)$ of transition times is given in term of $\psi_{0}(t \mid v)$ and $p_{m}(v)$

$$
\psi(t)=\int_{0}^{\infty} d v \frac{v p_{m}(v)}{\left\langle v_{m}\right\rangle} \psi_{0}(t \mid v) .
$$

For times $t \ll \tau_{D}$, it can be approximated by $\psi(t) \approx \ell_{v}^{2} t^{-3} p_{m}\left(\ell_{v} / t\right)$. For times larger than $\tau_{D}, \psi(t)$ decreases to 0 exponentially fast. Note that this result quantifies particle transition times from first principles, namely, pore-scale advection and diffusion, and network scale flow variability.

So far, we have considered motion along particle paths while our focus lies on dispersion in streamwise direction, that is, aligned with the mean pressure gradient. In analogy to purely advective motion, the increments $\ell_{v}$ along tortuous particle paths are projected onto the streamwise increments in terms of tortuosity $\chi$ such that the particle position $x(t)$ at time $t$ is given by $x(t)=s(t) / \chi$. In order to determine $\chi$, we note that, under ergodic flow conditions, the asymptotic mean particle velocity is equal to the mean flow velocity $\bar{u}$. This implies that, as particles sample a representative part of the spatial flow variability they assume asymptotically the mean flow velocity, which is equal to the Darcy velocity divided by porosity [9]. The stochastic time-domain random walk (TDRW) model 

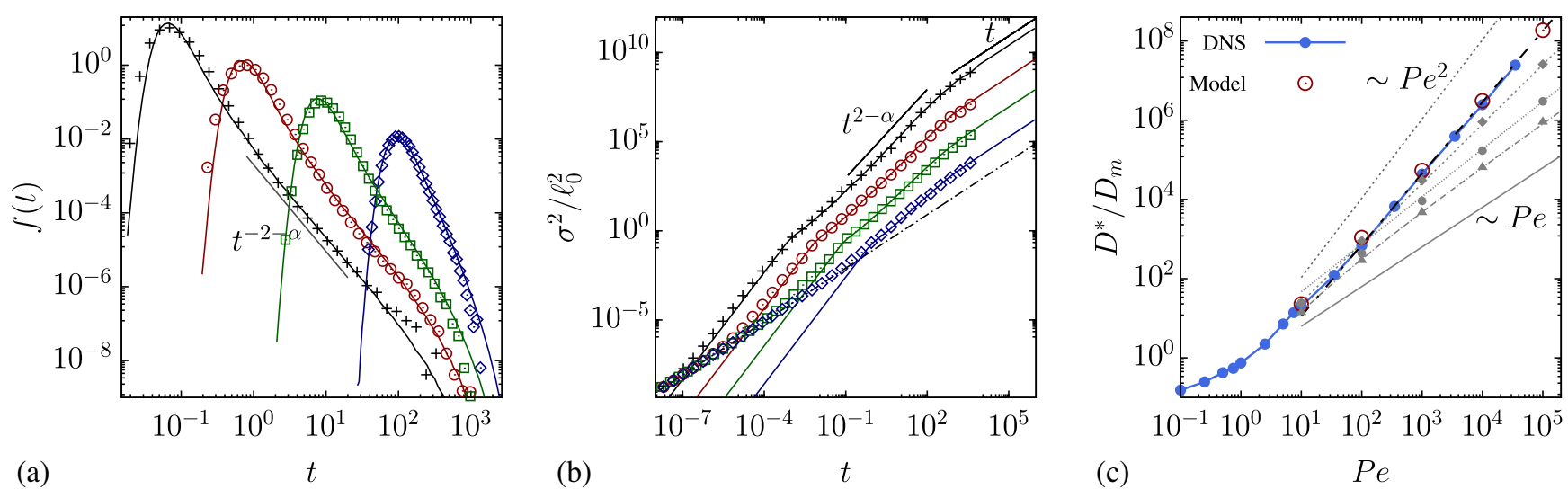

FIG. 3. (a) First arrival time PDFs at distance $x \approx 32 \ell_{0}$ and (b) $\sigma^{2}(t)$ for (crosses) $P e=10^{4}$, (circles) $10^{3}$, (squares) $10^{2}$, and (rhombs) 10. (c) Streamwise hydrodynamic dispersion coefficients versus Péclet number from (full circles) direct numerical simulations, and (empty circles) the model predictions. The gray symbols show the predictions for Gamma-distributed Eulerian speeds with exponents (top to bottom) $\alpha=0.5,0.8,1$, the corresponding lines show the theoretical scalings.

described by Eqs. (1)-(5) yields for the asymptotic average particle velocity $v_{\infty}=\ell_{v} / \chi\langle\tau\rangle \equiv \bar{u}$, where $\langle\tau\rangle$ is the average transition time [50]. This gives for tortuosity $\chi=\ell_{v} / \bar{u}\langle\tau\rangle$. Using Eqs. (5) and (4), we obtain the explicit expression

$$
\chi=\chi_{a}\left[1-\int_{0}^{\infty} d v \frac{1-\exp \left(-\mathrm{Pe}_{v}\right)}{\mathrm{Pe}_{v}} p_{m}(v)\right]^{-1} .
$$

The behavior of $\chi$ for the Berea sample is shown in Fig. 2(c). For Pe $\gg 1$ it tends to advective tortuosity $\chi_{a}$ and increases monotonically for decreasing Pe.

We use the derived theory to quantify and elucidate anomalous and normal hydrodynamic dispersion behaviors. To this end, we focus on speed PDFs that behave as power laws for speeds much smaller than the average, $p_{m}(v) \sim$ $v^{\alpha-1}$ with $0<\alpha \leq 1$. Such behaviors have been observed for a wide range of porous media [17,21,44]. Note that $\alpha=1$ for a channel or tube. For the Berea sample we find $\alpha \approx 0.35$ as shown in Fig. 2(a). The corresponding transition time PDF behaves as $\psi(t) \sim\left(t / \tau_{v}\right)^{-2-\alpha}$ for times $t \ll \tau_{D}$, and decays exponentially fast for $t \gg \tau_{D}$, which is a key feature of the impact of pore-scale mixing. To facilitate the analysis, we note that the stochastic TDRW model described by Eqs. (1)-(6) constitutes a CTRW so that we can use the CTRW machinery to derive the hydrodynamic dispersion behaviors predicted by the theory [34].

We focus on the PDF $f(t)$ of first arrival times at control planes perpendicular to the mean flow direction, and the longitudinal displacement variance $\sigma^{2}(t)=$ $\left\langle x(t)^{2}\right\rangle-\langle x(t)\rangle^{2}$. For times $t \ll \tau_{D}$ particles see only the power-law scaling $\psi(t) \sim t^{-2-\alpha}$ of the transition time PDF. In this regime, particle motion is history dependent because transition times may be of the order of the observation time. CTRW based on the generalized central limit theorem predicts power-law scaling as $f(t) \sim t^{-2-\alpha}$ [51]. The displacement variance is predicted to scale as $\sigma^{2}(t) \sim \bar{u} \ell_{v}\left(t / \tau_{v}\right)^{2-\alpha}$ for $0<\alpha<1$ and as $\sigma^{2}(t) \sim \bar{u} \ell_{v}\left(t / \tau_{v}\right) \ln \left(t / \tau_{v}\right)$ for $\alpha=1$ $[34,52]$. This means, dispersion is anomalous. For times $t \gg$ $\tau_{D}$ all particles are able to access the full spectrum of transition times and become statistically equal because the memory of the initial velocities is lost. This leads to an exponential cutoff in $f(t)$ [50], and linear (Fickian) scaling of $\sigma^{2}(t)$ as $2 D^{*} t$. The hydrodynamic dispersion coefficient $D^{*}$ can be obtained by matching the preasymptotic and asymptotic expressions for $\sigma^{2}(t)$ at $t=\tau_{D}$. This gives $D^{*} / D_{m} \sim \mathrm{Pe}^{2-\alpha}$. A similar behavior was obtained by Bijeljic et al. [20] based on empirical transition time PDFs using CTRW theory [50]. The theory presented here directly links these behaviors to the distribution of Eulerian flow speeds. For $\alpha=1$, we obtain $D^{*} / D_{m} \sim \mathrm{Pe} \ln (\mathrm{Pe})$, which is equivalent to the expression derived by Saffman [8] and Koch and Brady [31] based on the assumption that the distribution of flow speeds is flat, which is characteristic of the linear flow profile at pore walls. For $\alpha>1$, the theory predicts $D^{*} / D_{m} \sim$ Pe. Details are given in the Supplemental Material [34].

These features of anomalous and asymptotic Fickian dispersion are illustrated in Fig. 3 for the Berea sandstone sample. The power-law scaling and exponential tempering of $f(t)$ are shown in Fig. 3(a) for different Pe. Figure 3(b) shows the evolution of $\sigma^{2}(t)$. The theory predicts the early time ballistic behavior, the cross-over to anomalous dispersion for $t>\tau_{v}$, and the transition to normal dispersion for times $t>\tau_{D}$. The diffusive behavior at very early times $t<D_{m} / \bar{u}^{2}$ is not resolved by the theory because it does not explicitly represent (Brownian) particle motion at short times. The impact of diffusion is accounted for through its effect on particle transition times, tortuosity, and velocity sampling as detailed above. The theory predicts that the dependence of $D^{*}$ on Pe is constrained between $D^{*} / D_{m} \sim \mathrm{Pe}^{2}$ and $D^{*} / D_{m} \sim \mathrm{Pe}$. This is illustrated in Fig. 3(c), which shows $D^{*}$ versus Pe obtained from the Berea sample as well as the theoretical predictions for $\alpha=0.5,0.8,1$ using a Gamma-distributed $p_{e}(v)$. 
Data from a broad range of experimental and numerical studies of dispersion in a variety of porous media $[2,9,23,33,53,54]$ indicate a non-linear increase of $D^{*}$ with Pe. These data are often interpreted jointly by a single regression [2,9], which implicitly assumes the existence of a universal behavior across different types of porous media. The derived theory indicates that neither the Pe dependence of asymptotic hydrodynamic dispersion nor its evolution are universal, but depend on the flow distribution. On the other hand, it shows that dispersion can be predicted based on the distribution of flow speeds, which can be applied across a broad range of porous media.

The theory quantifies anomalous and normal hydrodynamic dispersion from first principles in terms of the characteristic velocity scale, the Eulerian flow speed, and pore-scale diffusion. It is valid for $\mathrm{Pe}>1$ and based on the knowledge of the Eulerian speed distribution. It is not constrained by transport measurements. The fundamental nature of the considered flow and transport processes in the conceptual picture of a network of conducts allows application of the key elements of the derived theory to transport of dissolved chemicals, bacteria, and colloids in a wide range of porous media also under non-Newtonian and multiphase flow conditions.

A.P. and M. D. gratefully acknowledge the support of the FP7 European Research Council (ERC) through the consolidator project MHetScale (617511) and the Spanish Ministry of Science and Innovation through the project HydroPore (PID2019-106887 GB-C31). The authors gratefully acknowledge the support of the CNRS-PICS project CROSSCALE (Project No. 280090).

*marco.dentz@csic.es

[1] H. Brenner and D. Edwards, Macrotransport Processes (Butterworth-Heinemann, MA, USA, 1993).

[2] M. Sahimi, Flow and Transport in Porous Media and Fractured Rock: From Classical Methods to Modern Approaches (John Wiley \& Sons, New York, 2011).

[3] A. J. Valocchi, D. Bolster, and C. J. Werth, Mixing-limited reactions in porous media, Transp. Porous Media 130, 157 (2019).

[4] Geological Storage of CO2 in Deep Saline Formations, edited by A. Niemi, J. Bear, and J. Bensabat (Springer, Netherlands, 2017).

[5] D. Maggiolo, F. Picano, F. Zanini, S. Carmignato, M. Guarnieri, S. Sasic, and H. Ström, Solute transport and reaction in porous electrodes at high Schmidt numbers, J. Fluid Mech. 896, A13 (2020).

[6] M. Berg, Y. Davit, M. Quintard, and S. Lorthois, Modelling solute transport in the brain microcirculation: is it really well mixed inside the blood vessels? J. Fluid Mech. 884, A39 (2019).

[7] G. de Josselin de Jong, Longitudinal and transverse diffusion in granular deposits, Trans., Am. Geophys. Union 39, 67 (1958).
[8] P. G. Saffman, A theory of dispersion in a porous medium, J. Fluid Mech. 6, 321 (1959).

[9] J. Bear, Dynamics of Fluids in Porous Media (American Elsevier, New York, 1972).

[10] M. B. Isichenko, Percolation, statistical topography, and transport in random media, Rev. Mod. Phys. 64, 961 (1992).

[11] J. P. Bouchaud and A. Georges, Anomalous diffusion in disordered media: Statistical mechanisms, models and physical applications, Phys. Rep. 195, 127 (1990).

[12] M. Moroni and J. H. Cushman, Statistical mechanics with three-dimensional particle tracking velocimetry experiments in the study of anomalous dispersion. II. experiments, Phys. Fluids 13, 81 (2001).

[13] M. Levy and B. Berkowitz, aMeasurement and analysis of non-Fickian dispersion in heterogeneous porous media, J. Contam. Hydrol. 64, 203 (2003).

[14] J. D. Seymour, J. P. Gage, S. L. Codd, and R. Gerlach, Anomalous fluid transport in porous media induced by biofilm growth, Phys. Rev. Lett. 93, 198103 (2004).

[15] V. L. Morales, M. Dentz, M. Willmann, and M. Holzner, Stochastic dynamics of intermittent pore-scale particle motion in three-dimensional porous media: Experiments and theory, Geophys. Res. Lett. 44, 9361 (2017).

[16] M. Carrel, V. L. Morales, M. Dentz, N. Derlon, E. Morgenroth, and M. Holzner, Pore-scale hydrodynamics in a progressively bioclogged three-dimensional porous medium: 3-d particle tracking experiments and stochastic transport modeling, Water Resour. Res. 54, 2183 (2018).

[17] M. Souzy, H. Lhuissier, Y. Méheust, T. L. Borgne, and B. Metzger, Velocity distributions, dispersion and stretching in three-dimensional porous media, J. Fluid Mech. 891, A16 (2020).

[18] P. Gouze, T. Le Borgne, R. Leprovost, G. Lods, T. Poidras, and P. Pezard, Non-Fickian dispersion in porous media: 1. multiscale measurements using single-well injection withdrawal tracer tests, Water Resour. Res. 44, W06426 (2008).

[19] R. Haggerty and S. M. Gorelick, Multiple-rate mass transfer for modeling diffusion and surface reactions in media with pore-scale heterogeneity, Water Resour. Res. 31, 2383 (1995).

[20] B. Bijeljic, P. Mostaghimi, and M. J. Blunt, Signature of Non-Fickian Solute Transport in Complex Heterogeneous Porous Media, Phys. Rev. Lett. 107, 204502 (2011).

[21] B. Bijeljic, A. Raeini, P. Mostaghimi, and M. J. Blunt, Predictions of non-fickian solute transport in different classes of porous media using direct simulation on porescale images, Phys. Rev. E 87, 013011 (2013).

[22] P. De Anna, T. Le Borgne, M. Dentz, A. M. Tartakovsky, D. Bolster, and P. Davy, Flow Intermittency, Dispersion, and Correlated Continuous Time Random Walks in Porous Media, Phys. Rev. Lett. 110, 184502 (2013).

[23] M. Icardi, G. Boccardo, D. L. Marchisio, T. Tosco, and R. Sethi, Pore-scale simulation of fluid flow and solute dispersion in three-dimensional porous media, Phys. Rev. E 90, 013032 (2014).

[24] P. K. Kang, P. de Anna, J. P. Nunes, B. Bijeljic, M. J. Blunt, and R. Juanes, Pore-scale intermittent velocity structure underpinning anomalous transport through 3-d porous media, Geophys. Res. Lett. 41, 6184 (2014). 
[25] D. W. Meyer and B. Bijeljic, Pore-scale dispersion: Bridging the gap between microscopic pore structure and the emerging macroscopic transport behavior, Phys. Rev. E 94, 013107 (2016).

[26] M. Li, T. Qi, Y. Bernabé, J. Zhao, Y. Wang, D. Wang, and Z. Wang, Simulation of solute transport through heterogeneous networks: analysis using the method of moments and the statistics of local transport characteristics, Sci. Rep. 8, 3780 (2018).

[27] Y. Liu and P. K. Kitanidis, Applicability of the dual-domain model to nonaggregated porous media, Groundwater 50, 927 (2012).

[28] M. Dentz, M. Icardi, and J. J. Hidalgo, Mechanisms of dispersion in a porous medium, J. Fluid Mech. 841, 851 (2018).

[29] A. Puyguiraud, P. Gouze, and M. Dentz, Upscaling of anomalous pore-scale dispersion, Transp. Porous Media 128, 837 (2019).

[30] A. Nissan and B. Berkowitz, Anomalous transport dependence on p'eclet number, porous medium heterogeneity, and a temporally varying velocity field, Phys. Rev. E 99, 033108 (2019).

[31] D. L. Koch and J. F. Brady, Dispersion in fixed beds, J. Fluid Mech. 154, 399 (1985).

[32] U. M. Scheven, Pore-Scale Mixing and Transverse Dispersivity of Randomly Packed Monodisperse Spheres, Phys. Rev. Lett. 110, 214504 (2013).

[33] B. Bijeljic and M. J. Blunt, Pore-scale modeling and continuous time random walk analysis of dispersion in porous media, Water Resour. Res. 42, W01202 (2006).

[34] See Supplemental Material at http://link.aps.org/ supplemental/10.1103/PhysRevLett.126.164501 for the setup of the direct numerical simulations, and detailed derivations, which includes Refs. [35-43].

[35] P. Gouze, Y. Melean, T. Le Borgne, M. Dentz, and J. Carrera, Non-fickian dispersion in porous media explained by heterogeneous microscale matrix diffusion, Water Resour. Res. 44, W11416 (2008).

[36] F. Gjetvaj, A. Russian, P. Gouze, and M. Dentz, Dual control of flow field heterogeneity and immobile porosity on non- Fickian transport in Berea sandstone, Water Resour. Res. 51, 8273 (2015).

[37] S. V. Patankar, Numerical Heat Transfer and Fluid Flow (Hemisphere Publishing Corporation, Washington, New York, 1980).

[38] H. G. Weller, G. Tabor, H. Jasak, and C. Fureby, A tensorial approach to computational continuum mechanics using object-oriented techniques, Comput. Phys. 12, 620 (1998).
[39] R. Guibert, P. Horgue, G. Debenest, and M. Quintard, A comparison of various methods for the numerical evaluation of porous media permeability tensors from pore-scale geometry, Math. Geosci. 48, 329 (2015).

[40] D. W. Pollock, Semianalytical computation of path lines for finite-difference models, Ground Water 26, 743 (1988).

[41] P. Mostaghimi, B. Bijeljic, M. Blunt et al., Simulation of flow and dispersion on pore-space images, SPE Journal 17, 1131 (2012).

[42] J. R. Michael, W. R. Schucany, and R. W. Haas, Generating random variates using transformations with multiple roots, Am. Stat. 30, 88 (1976).

[43] H. Scher and M. Lax, Stochastic transport in a disordered solid. I. Theory, Phys. Rev. B 7, 4491 (1973).

[44] A. Puyguiraud, P. Gouze, and M. Dentz, Stochastic dynamics of Lagrangian pore-scale velocities in threedimensional porous media, Water Resour. Res. 55, 1196 (2019).

[45] A. Koponen, M. Kataja, and J. Timonen, Tortuous flow in porous media, Phys. Rev. E 54, 406 (1996).

[46] B. Ghanbarian, A. G. Hunt, R. P. Ewing, and M. Sahimi, Tortuosity in porous media: A critical review, Soil Sci. Soc. Am. J. 77, 1461 (2013).

[47] M. Holzner, V. L. Morales, M. Willmann, and M. Dentz, Intermittent Lagrangian velocities and accelerations in threedimensional porous medium flow, Phys. Rev. E 92, 013015 (2015).

[48] P. De Anna, B. Quaife, G. Biros, and R. Juanes, Prediction of velocity distribution from pore structure in simple porous media, Phys. Rev. Fluids 2, 124103 (2017).

[49] K. Alim, S. Parsa, D. A. Weitz, and M. P. Brenner, Local Pore Size Correlations Determine Flow Distributions in Porous Media, Phys. Rev. Lett. 119, 144501 (2017).

[50] M. Dentz, A. Cortis, H. Scher, and B. Berkowitz, Time behavior of solute transport in heterogeneous media: transition from anomalous to normal transport, Adv. Water Resour. 27, 155 (2004).

[51] B. Berkowitz, A. Cortis, M. Dentz, and H. Scher, Modeling non-Fickian transport in geological formations as a continuous time random walk, Rev. Geophys. 44, RG2003 (2006).

[52] M. F. Shlesinger, Asymptotic solutions of continuous-time random walks, J. Stat. Phys. 10, 421 (1974).

[53] H. O. Pfannkuch, Contribution a l' 'etude des d'eplacements de fluides miscibles dans un milieux poreux, Rev. Inst. Fr. Petr. 18, 215 (1963).

[54] B. Bijeljic, A. H. Muggeridge, and M. J. Blunt, Pore-scale modeling of longitudinal dispersion, Water Resour. Res. 40 (2004). 